The University of Southern Mississippi

The Aquila Digital Community

Faculty Publications

$2-1-2000$

\title{
Characteristics of Driven Polymer Surfaces: Growth and Roughness
}

Grace M. Foo

National University of Singapore

Ras B. Pandey

University of Southern Mississippi, ras.pandey@usm.edu

Follow this and additional works at: https://aquila.usm.edu/fac_pubs

Part of the Physics Commons

\section{Recommended Citation}

Foo, G. M., Pandey, R. B. (2000). Characteristics of Driven Polymer Surfaces: Growth and Roughness.

Physical Review E, 61(2), 1793-1799.

Available at: https://aquila.usm.edu/fac_pubs/4314

This Article is brought to you for free and open access by The Aquila Digital Community. It has been accepted for inclusion in Faculty Publications by an authorized administrator of The Aquila Digital Community. For more information, please contact Joshua.Cromwell@usm.edu. 


\title{
Characteristics of driven polymer surfaces: Growth and roughness
}

\author{
Grace M. Foo ${ }^{1}$ and R. B. Pandey ${ }^{2}$ \\ ${ }^{1}$ Supercomputing and Visualization Unit, Computer Center, National University of Singapore, Singapore 119260 \\ ${ }^{2}$ Department of Physics and Astronomy, University of Southern Mississippi, Hattiesburg, Mississippi 39406-5046
}

(Received 27 January 1999)

\begin{abstract}
Using a Monte Carlo simulation, the growth and roughness characteristics of polymer surfaces are studied in $2+1$ dimensions. Kink-jump and reptation dynamics are used to move polymer chains under a driving field where they deposit onto an impenetrable attractive wall. Effects of field $(E)$, chain length $\left(L_{c}\right)$, and the substrate size $(L)$ on the growing surfaces are studied. In low field, the interface width $(W)$ shows a crossover from one power-law growth in time $\left(W \sim t^{\beta_{1}}\right)$ to another $\left(W \sim t^{\beta_{2}}\right)$, before reaching its asymptotic value $\left(W_{s}\right)$, with $\beta_{1}(\sim 0.5 \pm 0.1)<\beta_{2}(\sim 0.6-1.0)$. For short chain lengths $\left(L_{c}=4\right)$, the saturated width $\left(W_{s}\right)$ is independent of the substrate length $(L)$, while for long chain lengths, $W_{s}$ decays with $L$ before becoming independent at large $L . W_{s}$ depends strongly on the magnitude of the field: for short chains, $W_{s} \sim E^{-\delta}$ with $\delta \simeq 0.4$, while for long chains, it varies nonmonotonically with $E$.
\end{abstract}

PACS number(s): 68.35.Ct, 81.05.Lg, 81.15.Pq

\section{INTRODUCTION}

Growth processes and the characteristics of growing surfaces (or interfaces) have been the focus of much research due to their wide applications: in biology/biological systems, and in industries such as semiconductor, coatings, and thin films, etc. [1-11]. If species/particulates of type $A$ deposit on a substrate with a certain mechanism (dynamics), their density and the height of the growing surface of $A$ increases with time. The surface thickness or width $W$ of the growing surface, a commonly used measure of surface roughness, is defined as the variance of the height:

$$
\begin{gathered}
W^{2}=\sum_{i j}\left(h_{i j}-\bar{h}\right)^{2}, \\
\bar{h}=\sum_{i j} h_{i j} / N_{s},
\end{gathered}
$$

where $h_{i j}$ is the surface height at location $(i, j)$ on the wall/ substrate, and $\bar{h}$ is the mean surface height averaged over the surface with $N_{s}$ sites. In many theoretical models and experiments with particle systems [1], the surface width is found to obey scaling relations in time $(t)$ and substrate length $(L)$ :

$$
\begin{gathered}
W \sim t^{\beta}, \quad t \ll L^{z}, \\
W_{s} \sim L^{\alpha}, \quad t \gg L^{z},
\end{gathered}
$$

where $\beta$ is the growth exponent, $\alpha$ the roughness exponent, and $z=\alpha / \beta$ the dynamical scaling exponent. $W_{s}$, an asymptotic (saturated) value for the width, is approached after a sufficiently long time. Growth of the surface height $h$ may be described by a Langevin-type (Burgers) equation, known as the Kardar-Parisi-Zhang (KPZ) equation,

$$
\frac{\partial h}{\partial t}=\nu \nabla^{2} h+\frac{\lambda}{2}(\nabla h)^{2}+\eta
$$

With just the noise term $(\eta)$, we have a random deposition (RD) model in which the height is uncorrelated with the growth exponent $\beta=1 / 2$ ( $\alpha$ undetermined) [1]. A solution for the linear form $(\lambda=0)$, obtained by Edwards and Wilkinson (EW) [1,2], yields exponents $\alpha=(2-d) / 2$ and $\beta=(2-d) / 4$ for a $d$-dimensional interface. This equation describes a random deposition with surface relaxation via diffusion, the first term in Eq. (5). Kardar, Parisi, and Zhang analyzed the nonlinear (KPZ) equation (5) using renormalization-group and mapping techniques [3] and found a new universality class for the growth of the interface width with exponents $\alpha=1 / 2$ and $\beta=1 / 3$ for $d=1$. Various numerical solutions estimate $\beta \sim 0.24$ for $d=2$ [7]. The KPZ equation may describe various growth models such as ballistic deposition (BD), restricted solid-on-solid, and Eden models. Numerous other models that include other features, such as surface diffusion and nonlocal effects, have been explored [1-11]. Some of these give different surface morphologies and belong to new universality classes. In simulations and experiments with nonequilibrium growth, where an external field drives the particles [Eq. (5) with a force term], the surfaces have high growth exponents $(\beta \rightarrow 1.0)$ [8,9]. High growth exponents are also seen for driven growth in porous media $[10,11]$.

Much less is known of the processes and characteristics of growing surfaces arising from extended objects like polymer chains [12]. In this paper, we present a Monte Carlo simulation study of a growth model for driven polymer chains on a discrete lattice. Polymer chains are released continuously at one end of a lattice and move under the influence of an external field toward an impenetrable attractive wall at the opposite end where they deposit, as described in Sec. II. Simulation results, growth and roughness characteristics of the growing surface, are discussed in Sec. III.

\section{METHOD AND MODEL}

We consider discrete three-dimensional lattices of size $L_{x} \times L \times L$ with comparatively large $L_{x}$, typically $L_{x}$ $=100-200$, and $L=20-80$. Polymer chains of length $L_{c}$ 


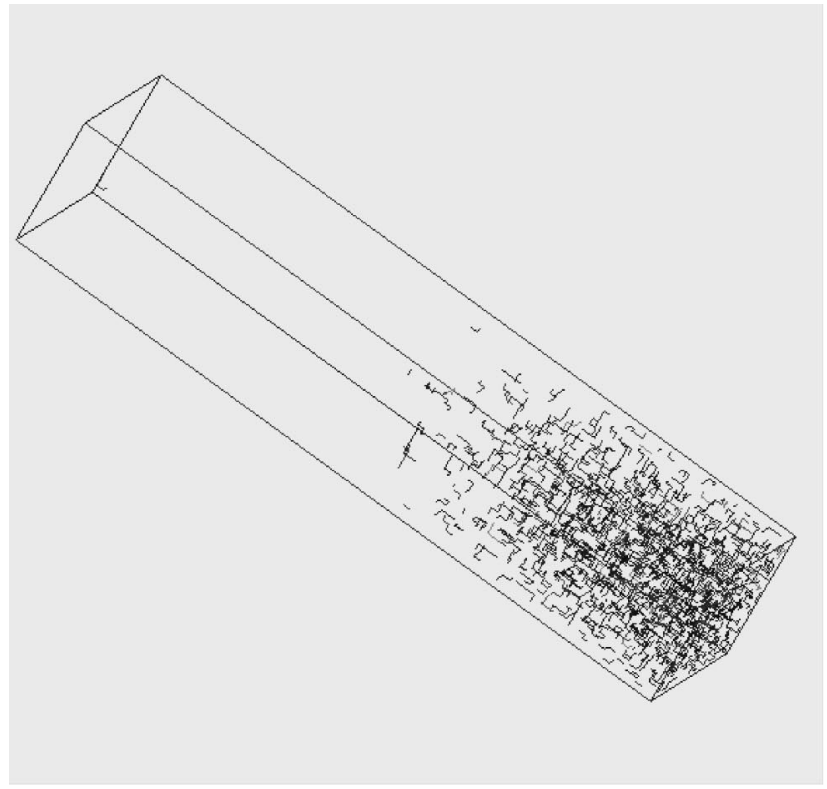

(a)

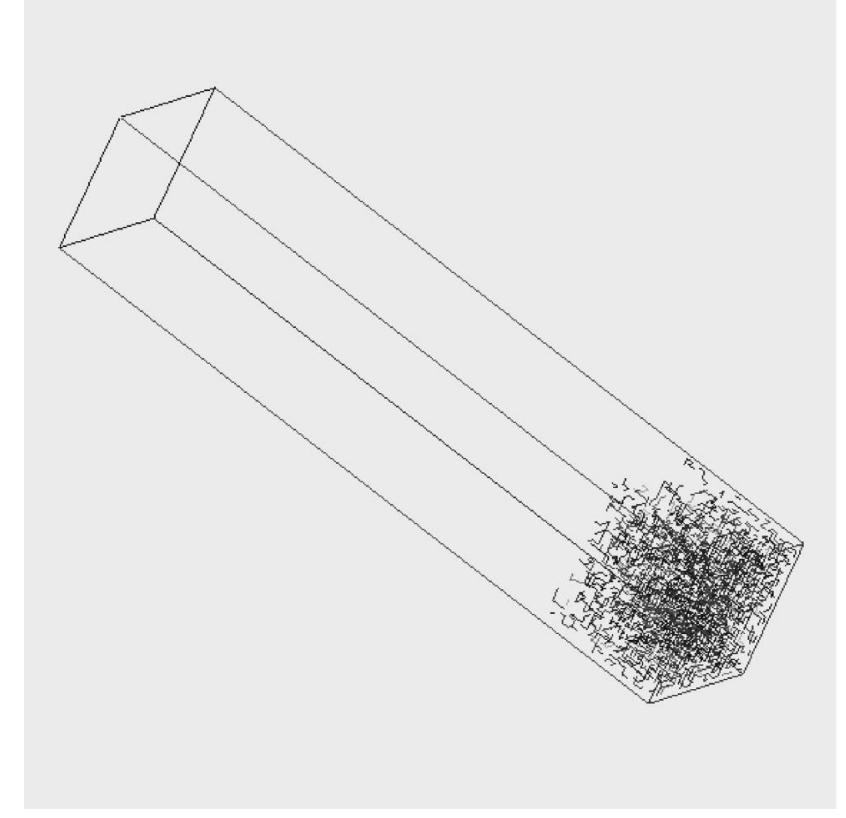

(b)

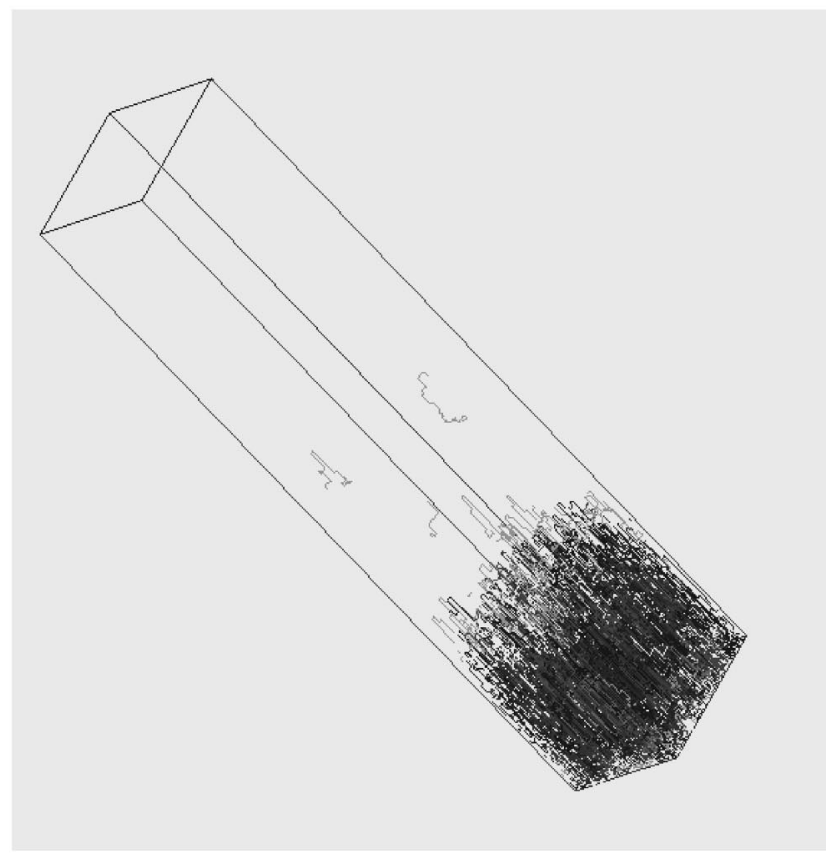

(c)

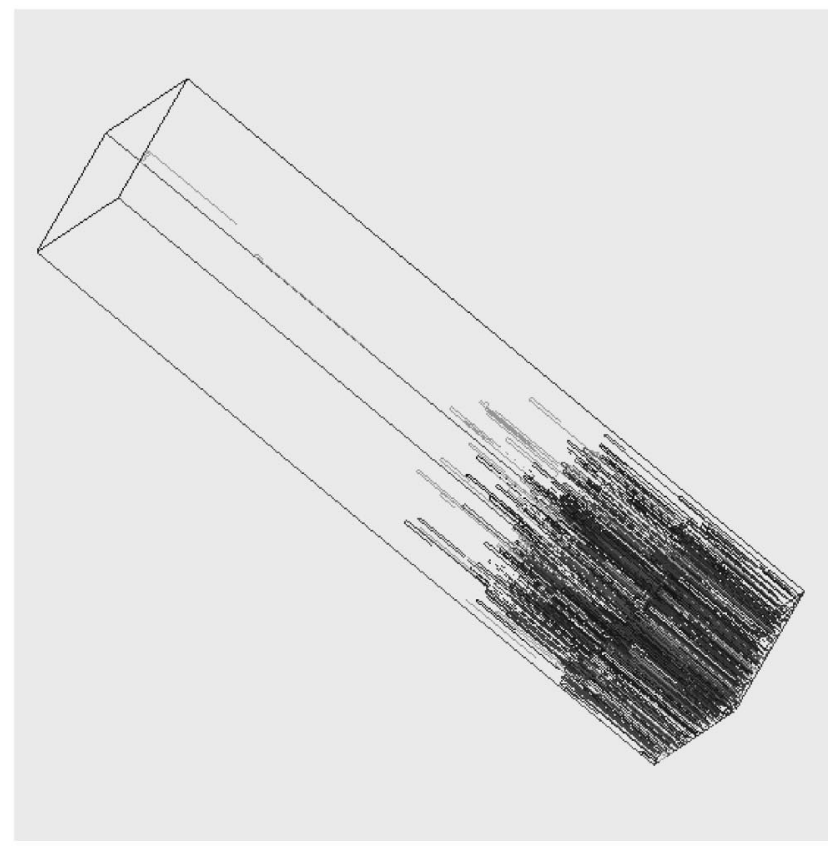

(d)

FIG. 1. Snapshots of chains of length $L_{c}=4$ at $80000 \mathrm{MCS}$, in field of magnitude $E=0.1$ (a), $E=0.5$ (b), $L_{c}=40$ at $E=0.5$ (c), and $E=5.0(\mathrm{~d})$.

(with $L_{c}+1$ nodes) connected by constant bonds of unit length are modeled as self-avoiding walks (SAWs), with reptation and kink-jump dynamics [13-17] in a Metropolis framework. A small number of chains (1-8) are released at constant intervals [200 Monte Carlo steps (MCS)] from near the source end (i.e., $x=1$ to $x=0.2 L x$ ) of the lattice. They move toward an impenetrable attractive wall ( $y z$ plane) at the opposite end $\left(x=L_{x}\right)$ in the presence of a biased external field of strength $E$ in the longitudinal, positive $x$ direction. The interaction energy among the various components in- cluding the coupling of field with displacement are described next.

In addition to excluded volume, a nearest neighbor polymer-polymer repulsive and a polymer-wall attractive (adsorbing) interaction are implemented. For this, we assign a positive charge or interaction density $(\rho=1)$ to each chain node, and a negative charge $(\rho=-1)$ at each lattice position on the wall $\left(y z\right.$ plane at $\left.x=L_{x}\right)$. The external field couples with the movement of the chain nodes via interaction energy, $-E d x$, where $d x(=0$ or \pm 1$)$ is the displacement step along 

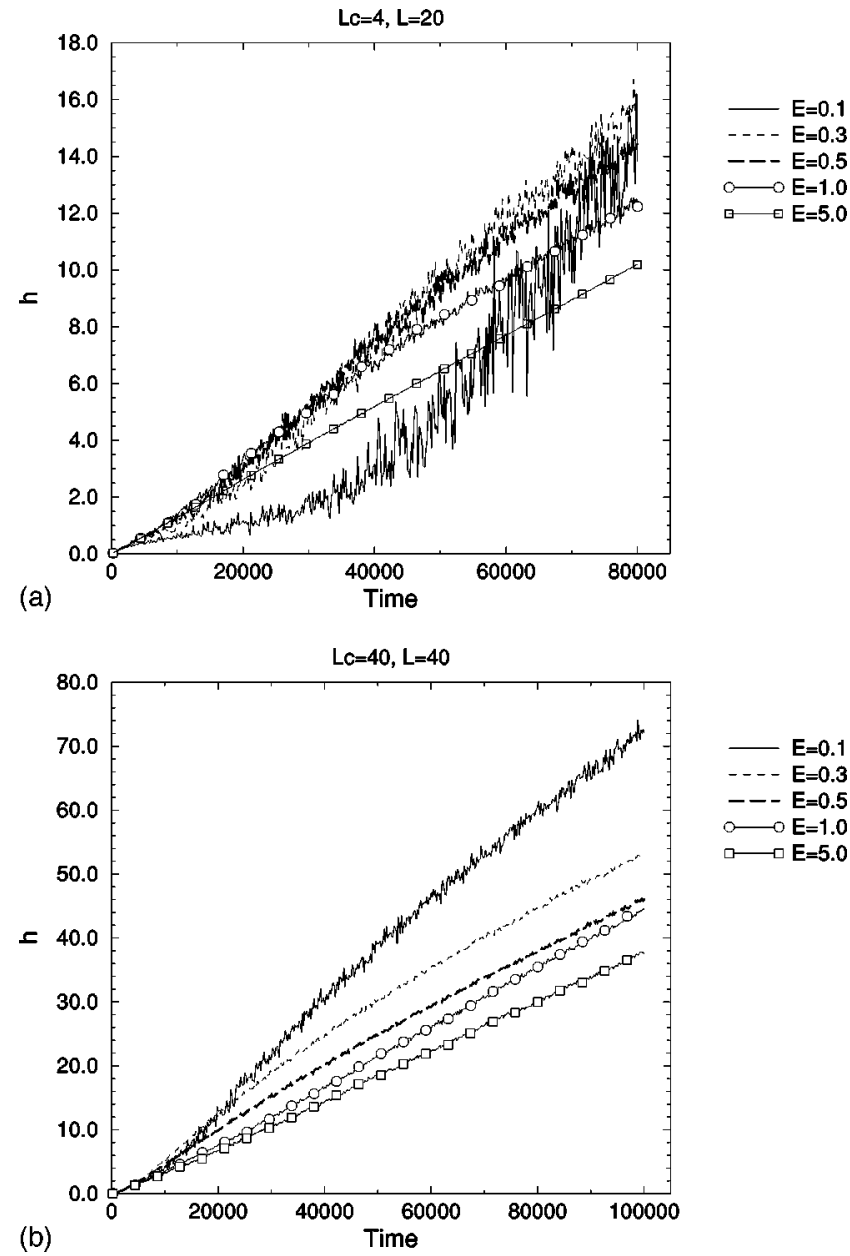

FIG. 2. Time variation of surface height for chains of length $L_{c}=4$ (a), and $L_{c}=40$ (b) under various magnitudes of field.

the $x$ direction. Thus, the interaction energy of the system is described by

$$
E_{\mathrm{int}}=\sum_{i j} \rho_{i} \rho_{j}+E \sum_{i} \rho_{i} d x_{i}
$$

where the first summation is restricted to nearest neighbor sites, and $d x_{i}=0, \pm 1$ is the displacement of the $i$ th chain node along the $x$ direction. The Metropolis Monte Carlo algorithm is used to move a randomly selected node. A periodic boundary condition is used across the $y$ and $z$ boundaries. One MCS is defined as $N\left(=L_{x} \times L \times L\right)$ attempts to move randomly selected chain nodes. In the random selection of chain nodes, kink-jump moves are attempted if internal nodes are selected while reptation or end-flip moves are attempted with equal probability if end nodes are selected. In an earlier model study of growing chains [18], we used only reptation moves and did not attempt to compute surface growth characteristics. Inclusion of kink-jump moves makes the model more realistic.

Simulations are performed with a range of chain lengths $\left(L_{c}=4,20,40,60\right.$, and 80), field strengths $(E=0.1,0.3,0.5$, 1 , and 5), and system sizes ( $L=20,30,40,50,60$, and 80$)$ at a fixed temperature $T=1$. Units for the interaction energy [Eq. (6)], field strength $(E)$, and temperature $(T)$ are arbitrary and normalized by $k_{B}$, the Boltzmann constant. For

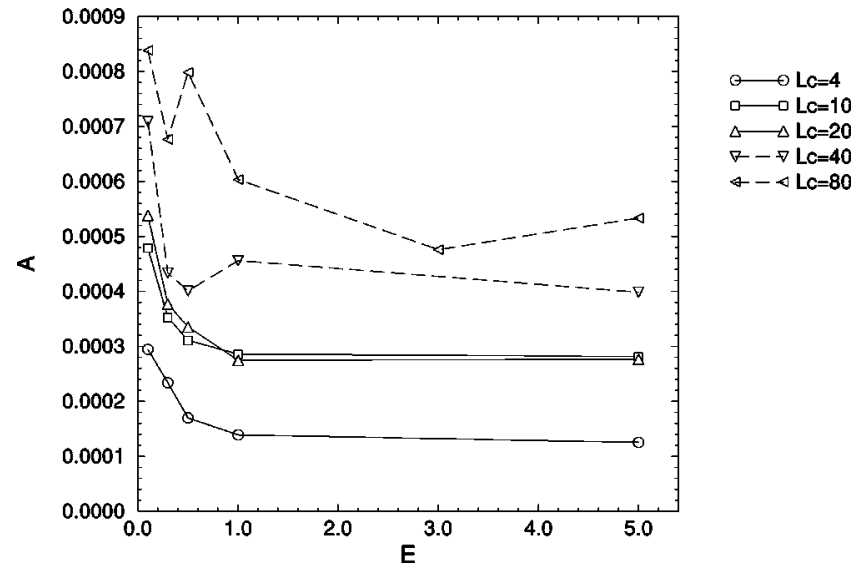

FIG. 3. $A$ versus $E$ for chains of various lengths.

each set of parameters, 1-7 simulations were performed to obtain reliable estimates of the surface width.

\section{RESULTS}

\section{A. Snapshots and surface height}

Visual inspection of snapshots of the chains provides a useful qualitative picture of their shape and that of the growing surface. We examine a few of these at the end of the simulation run. Figure 1 shows snapshots for short $\left(L_{c}=4\right)$ chains at different field magnitudes. At a low field $E=0.1$ [Fig. 1(a), the chains are less densely packed at the surface and in the bulk, i.e., between the wall and the growing surface] than at higher field $E=0.5$ [Fig. 1(b)]. Chains are packed even more closely in very high field $(E=5.0)$. A similar trend is seen in longer length chains. These longer chains $\left(L_{c}=40\right)$ are more noticeably aligned even at not too high fields $(E=0.5)$ [Fig. 1(c)]. Furthermore, there is considerably more layering and stacking of chains at very high field [Fig. 1(d)] compared with short chains. The surface is very rough since there are fewer chains in the outermost (surface) layer. For longer chains $\left(L_{c}=80\right)$, even a weaker field $(E=0.5)$ is sufficient for the onset of stacking (layering).

The mean height of the polymer surface [Eq. (2)] shows a nearly linear growth except at low field (Fig. 2). The surface

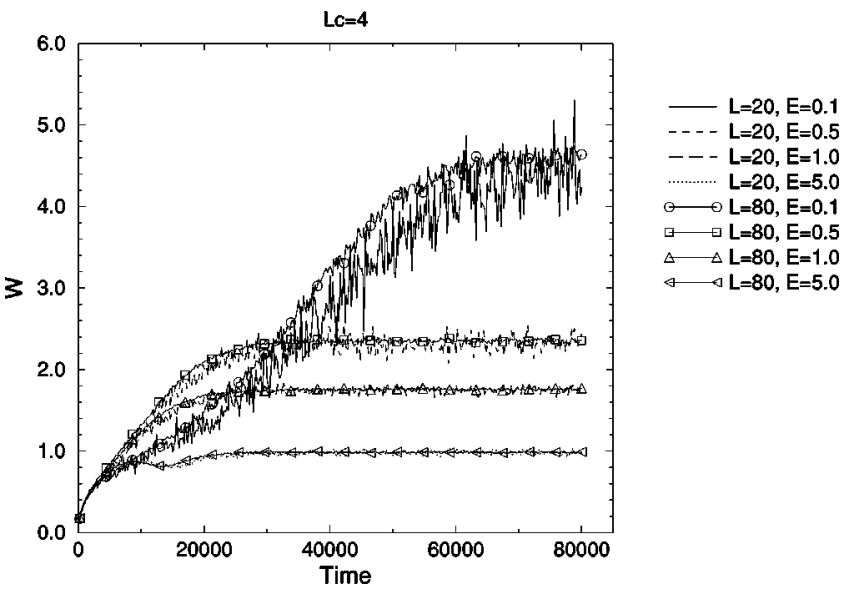

FIG. 4. Time variation of surface width for chains of length $L_{c}=4$ at various field magnitudes, $E=0.1$ to $E=5.0$. 


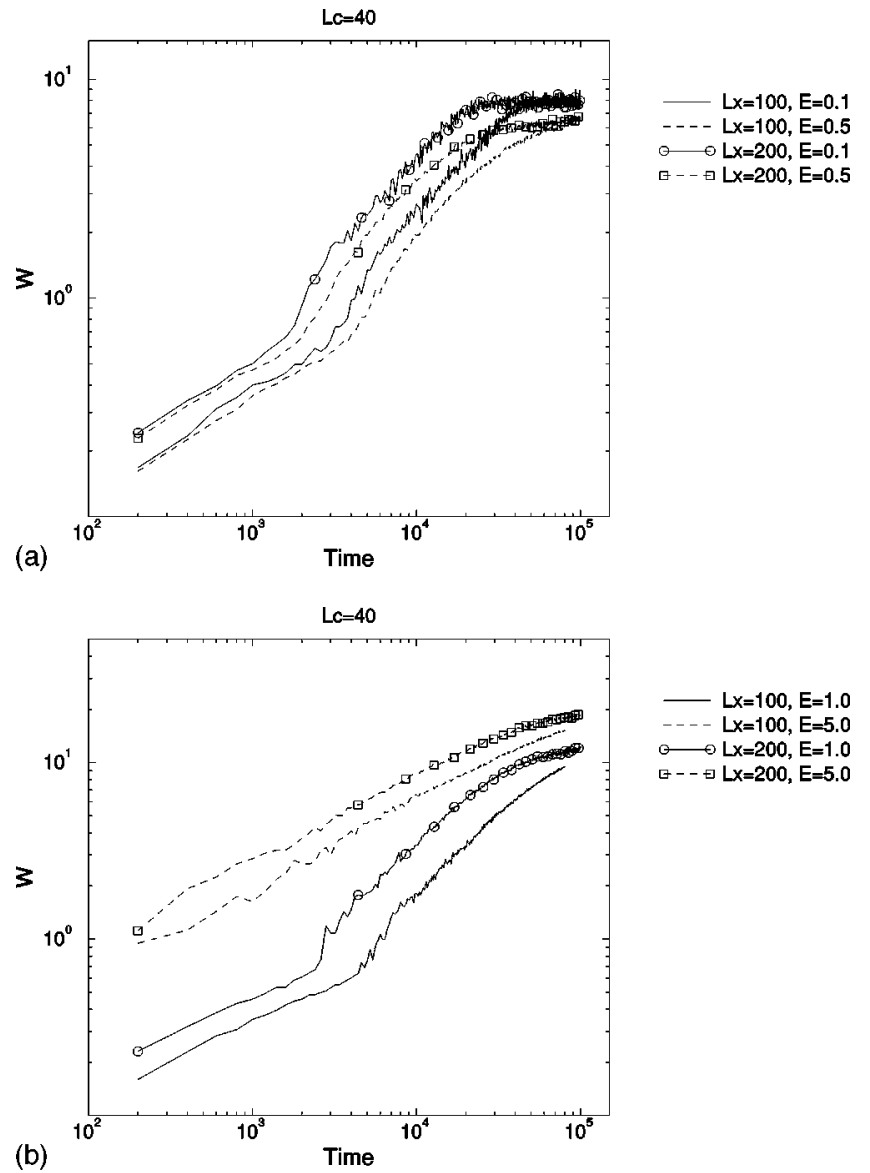

FIG. 5. Log-log plots of time variation of surface width for chains of length $L_{c}=40$ at $E=0.1,0.5$ (a), and $E=1.0,5.0$ (b).

height appears to relax faster with longer chains. Because of the polymer-polymer repulsion and steric constraints, the screening length (for the incoming chains to penetrate the surface) increases with the chain length. As a result, the average height of the surface grows faster with longer chain lengths. For a linear growth of the surface height,

$$
\bar{h}=A(E) t,
$$

we can analyze the variation of the growth rate $A(E)$ as a function of field as shown in Fig. 3. Note that the growth of the surface height is very sensitive to field in the low field regime and becomes nearly independent of $E$ in large field. In the low field regime, the growth rate $A(E)$ decays with the magnitude of the field. From these data, it is rather difficult to provide a quantitative empirical relation between $A$ and $E$, although a power-law decay cannot be ruled out.

\section{B. Growth of the surface width}

The width of the polymer surface/interface [Eq. (1)] increases over time and eventually saturates. Relaxation time to saturated width for short chains $\left(L_{c}=4\right)$ decreases as the field increases over the range of $E$ observed (Fig. 4). However, for longer chains $\left(L_{c}>20\right)$, there is a nonmonotonic variation in the relaxation of the width with increasing field. Relaxation time decreases as the field increases up to a point, but increases as the field increases further. The relaxation of the surface width for longer chains also seems to be sensitive
TABLE I. Growth exponent $\beta$ for shorter chains. As there is too much data, only detailed values for $L_{c}=4$ are shown and a range is shown for the others. Exponents slightly larger than 1 are truncated to 1 . Error bar $\sim 0.03$.

\begin{tabular}{|c|c|c|c|c|}
\hline \multirow{2}{*}{$\begin{array}{c}\text { Chain length } \\
L_{c}\end{array}$} & \multirow{2}{*}{$\begin{array}{c}\text { Field } \\
E\end{array}$} & \multirow{2}{*}{$\begin{array}{c}\text { Lattice size } \\
\qquad L\end{array}$} & \multicolumn{2}{|c|}{ Exponent } \\
\hline & & & $\beta_{1}$ & $\beta_{2}$ \\
\hline 4 & 0.1 & 20 & 0.58 & 1.0 \\
\hline 4 & 0.1 & 30 & 0.45 & 1.0 \\
\hline 4 & 0.1 & 40 & 0.46 & 1.0 \\
\hline 4 & 0.1 & 50 & 0.46 & 1.0 \\
\hline 4 & 0.1 & 80 & 0.40 & 1.0 \\
\hline 4 & 0.3 & 20 & 0.48 & 0.90 \\
\hline 4 & 0.3 & 30 & 0.42 & 0.82 \\
\hline 4 & 0.3 & 40 & 0.51 & 0.81 \\
\hline 4 & 0.3 & 50 & 0.51 & 0.81 \\
\hline 4 & 0.3 & 80 & 0.48 & 0.83 \\
\hline 4 & 0.5 & 20 & 0.53 & 0.77 \\
\hline 4 & 0.5 & 30 & 0.54 & 0.81 \\
\hline 4 & 0.5 & 40 & 0.60 & 0.75 \\
\hline 4 & 0.5 & 50 & 0.52 & 0.73 \\
\hline 4 & 0.5 & 80 & 0.45 & 0.75 \\
\hline 4 & 1.0 & 20 & 0.48 & 0.62 \\
\hline 4 & 1.0 & 30 & 0.49 & 0.61 \\
\hline 4 & 1.0 & 40 & 0.45 & 0.66 \\
\hline 4 & 1.0 & 50 & 0.50 & 0.59 \\
\hline 4 & 1.0 & 80 & 0.45 & 0.59 \\
\hline 4 & 5.0 & 20 & 0.45 & \\
\hline 4 & 5.0 & 30 & 0.43 & \\
\hline 4 & 5.0 & 40 & 0.43 & \\
\hline 4 & 5.0 & 50 & 0.45 & \\
\hline 4 & 5.0 & 80 & 0.42 & \\
\hline 4 & $0.1-5.0$ & $20-80$ & $0.40-0.60$ & $0.59-1.0$ \\
\hline 20 & $0.1-5.0$ & $20-80$ & $0.40-0.57$ & $0.64-0.96$ \\
\hline
\end{tabular}

to the lateral lattice size $L_{x}$, especially at high field. For fast relaxation, of the interface width, the value of $L_{x}$ must be large enough to accomodate a fully stretched out chain, i.e., the relaxed chain in field. At smaller $L_{x}$, chains may not relax fully before their deposition.

Examination of $\log -\log$ plots of the width with time (Fig. $5)$ for fields of low to medium strength $(E \leqslant 1.0)$ reveals two regions of power-law behavior, with a crossover in between. Growth of the interface width is described by the power-law exponents $\beta_{1} \sim 0.40-0.60$ (with most data points lying between $0.4-0.5)$ in the short time regime followed by a crossover to a faster growth with exponents $\beta_{2} \sim 0.58-1.0$ (Tables I and II), before it finally saturates in the long time regime. In contrast, at high field $(E \sim 5.0)$, the late powerlaw regime vanishes and only one power-law regime persists with exponents $\beta_{1} \sim 0.42-0.53$. We do not observe any trend/dependence of $\beta$ on chain length $L_{c}$ or field strength $E$, perhaps due to the limited quality of the data.

The exponent values in the first power-law regime $\left(\beta_{1}\right.$ $\sim 0.4-0.6)$ fall mostly in between the $\mathrm{KPZ}(\beta=0.24)$ and random deposition $(\beta=1 / 2)$ models. As in the KPZ model, some nonlocal effects are allowed in our chain growth model but we do not restrict development of overhangs and chains are allowed to move on the surface. In the short time regime 
TABLE II. Growth exponent $\beta$ for longer chains. As there is too much data, only detailed values for $L_{c}=40$ are shown and a range is shown for the others. Exponents slightly larger than 1 are truncated to 1 . Error bar $\sim 0.03$

\begin{tabular}{|c|c|c|c|c|}
\hline \multirow{2}{*}{$\begin{array}{c}\text { Chain length } \\
\qquad L_{c}\end{array}$} & \multirow{2}{*}{$\begin{array}{l}\text { Field } \\
\qquad E\end{array}$} & \multirow{2}{*}{$\begin{array}{c}\text { Lattice size } \\
\quad L\end{array}$} & \multicolumn{2}{|c|}{ Exponent } \\
\hline & & & $\beta_{1}$ & $\beta_{2}$ \\
\hline 40 & 0.1 & 40 & 0.44 & 0.83 \\
\hline 40 & 0.1 & 50 & 0.44 & 0.85 \\
\hline 40 & 0.1 & 60 & 0.46 & 0.76 \\
\hline 40 & 0.1 & 80 & 0.40 & 0.80 \\
\hline 40 & 0.3 & 40 & 0.41 & 0.87 \\
\hline 40 & 0.3 & 50 & 0.47 & 0.89 \\
\hline 40 & 0.3 & 60 & 0.45 & 1.0 \\
\hline 40 & 0.3 & 80 & 0.46 & 0.98 \\
\hline 40 & 0.5 & 40 & 0.42 & 0.76 \\
\hline 40 & 0.5 & 50 & 0.47 & 0.89 \\
\hline 40 & 0.5 & 60 & 0.47 & 1.0 \\
\hline 40 & 0.5 & 80 & 0.47 & 1.0 \\
\hline 40 & 1.0 & 40 & 0.40 & 0.85 \\
\hline 40 & 1.0 & 50 & 0.45 & 0.88 \\
\hline 40 & 1.0 & 60 & 0.46 & 0.91 \\
\hline 40 & 1.0 & 80 & 0.46 & 0.90 \\
\hline 40 & 5.0 & 40 & 0.43 & \\
\hline 40 & 5.0 & 50 & 0.48 & \\
\hline 40 & 5.0 & 60 & 0.47 & \\
\hline 40 & 5.0 & 80 & 0.48 & \\
\hline 40 & $0.1-5.0$ & $40-80$ & $0.40-0.48$ & $0.76-1.0$ \\
\hline 60 & $0.1-5.0$ & $60-80$ & $0.38-0.50$ & $0.76-0.97$ \\
\hline 80 & $0.1-5.0$ & $60-80$ & $0.41-0.50$ & $0.77-0.97$ \\
\hline
\end{tabular}

with lower field, chains are not fully relaxed and nodes are relatively independent (somewhat similar to particle deposition) with a local excluded volume effect. Thus, the exponent $\beta_{1}$ in our model falls close to the random deposition value of 0.5 .

In the long time regime, on the other hand, the chains have relaxed sufficiently to feel the effects of the field $(E \leqslant 1.0)$. We observe a second region of power-law growth for the surface width with high exponent values, $\beta_{2}$ $\sim 0.6-1.0$, which seems to indicate some sort of directed (anisotropic) growth. For example, Keblinski et al. [8] have observed a similar crossover to higher growth exponent in their simulations of driven particle growth in $(1+1)$ dimensions. They observed column like structures in the high growth regime. In our polymer model, we also observe a type of columnar growth (layered brushes) as the chains stretch out under influence of the field. The straightening effect of the field on a chain is tempered by pinning (steric hindrance) influences of other chains. The high growth exponents seem to signify a depinning of the chains and their rapid alignment by the field. We also note a small but noticeable dependence on $L_{c}$ in the growth exponent. The lower limit in $\beta_{2}$ increases progressively as the chain length increases, from $\beta_{2} \sim 0.59$ for $L_{c}=4$ to $\beta_{2} \sim 0.77$ for $L_{c}$ $=80$. The depinning of the chains and rapid growth in surface width is sensitive to the field. It seems to take place only at lower values of field; at higher fields (perhaps up to an optimal value of field) chains seem to show strong confor-

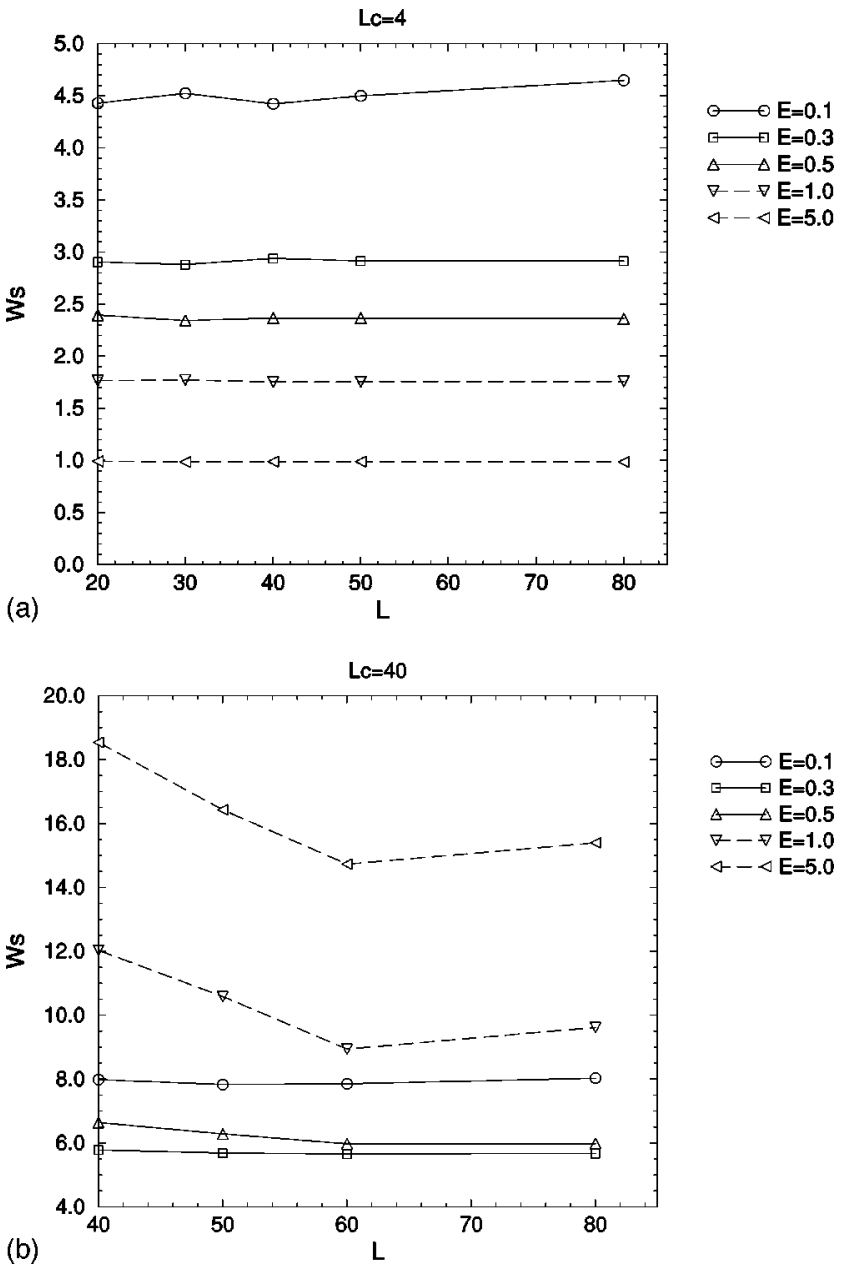

FIG. 6. Variation of saturated width $W_{s}$ with lattice size $L$ for chains of length $L_{c}=4$ (a), and $L_{c}=40$ (b).

mational stretching, and there is less variation in height. Thus, at high fields $(E=5.0)$, the width grows as a power law of time with exponents $\beta \sim 0.42-0.53$ and we observe no crossover to high exponent values.

\section{Saturated width and roughness}

The simulations are performed at least until the interface width saturates. The saturated width $W_{s}$ reflects the asymptotic roughness of the surface. Unlike some particle growth models [1-3], we do not observe a power-law scaling of the saturated width $W_{s}$ with the lattice size $L$ [Eq. (4)]. In fact, the saturated width for short chains $\left(L_{c}=4\right)$ does not appear to vary much with $L$ [Fig. 6(a)]. This implies that for short chains the roughness exponent is zero which may be for reasons yet to be fully understood. The power-law dependence of $W_{s}$ with the substrate length $(L)$ is due to finite size scaling when the height-height correlation length $\xi_{h} \geqslant L . \xi_{h}$ may depend on field and possibly on chain length. It is rather difficult to provide a complete multi parameter scaling for $W_{s}$ with parameters $\xi_{h}$, radius of gyration of chains $\left(R_{g}\right)$, and field $(E)$. The saturated width for longer chains $\left(L_{c}\right.$ $=20,40$ ), over a range of high $E$, seems to decrease linearly as $L$ increases [Fig. 6(b)]; we speculate $\xi_{h}<R_{g}<L$.

Variation of $W_{s}$ with $E$ shows some interesting behavior that seems to depend on the chain length. For short $L_{c}=4$ 


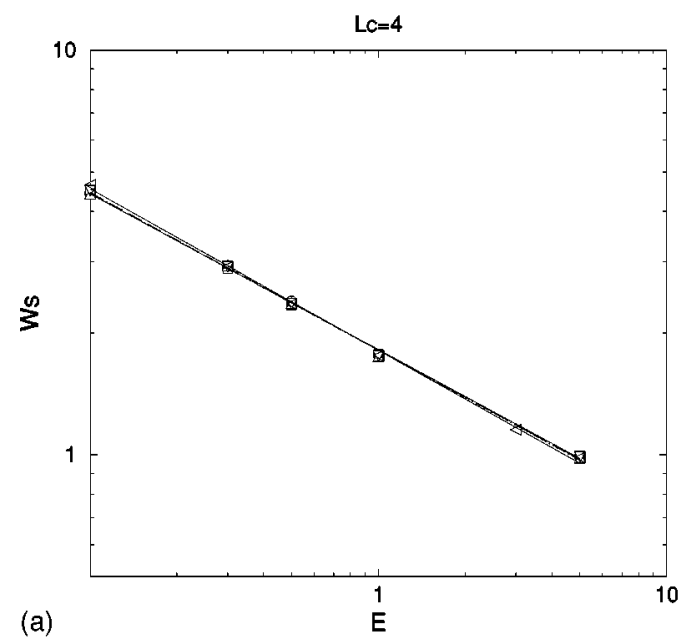

$$
\begin{aligned}
& -\mathrm{L}=20 \\
& \square \mathrm{L}=30 \\
& \Delta \mathrm{L}=40 \\
& \nabla \mathrm{L}=50 \\
& \triangleleft \mathrm{L}=80
\end{aligned}
$$

(a)
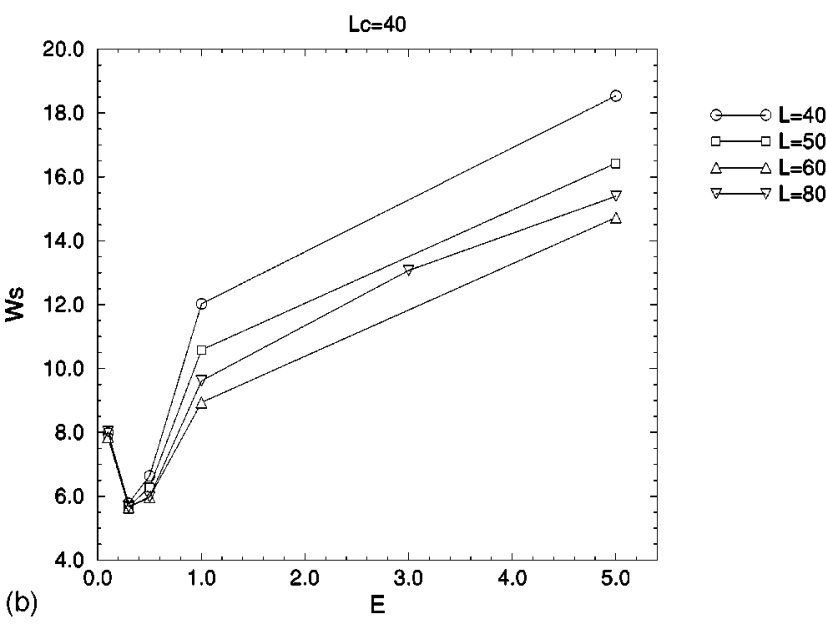

$\square \mathrm{L}=50$ $\triangle \mathrm{L}=60$ $\checkmark \mathrm{L}=80$

(b)

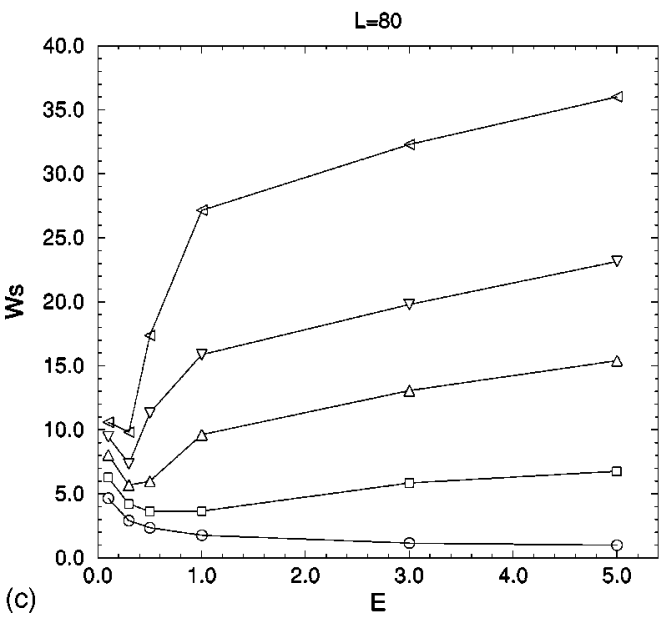

$\odot \mathrm{LC}=4$ $\square \longrightarrow \mathrm{LC}=20$ $\square \mathrm{LC}=40$ $\nabla \mathrm{Lc}=60$ $\leftrightarrow L C=80$

FIG. 7. Variation of saturated width $W_{s}$ with field $E$ for chains of length $L_{c}=4$ (log-log plot) (a), $L_{c}=40$ (b), and $W_{s}$ versus $E$ for various chain lengths at lattice size $L=80$ (c).

chains, it shows a power-law decrease as $E$ increases, i.e., $W_{s} \sim E^{-\delta}$ with exponent $\delta \sim 0.39-0.4$ [Fig. 7(a)]. The saturated width for longer chains $\left(L_{c}=20,40,80\right)$, on the other hand, varies nonmonotonically with $E$ [Fig. 7(b)]. It first decreases with increasing $E$ over a range of low $E$, as for short chains above. However, as $E$ increases beyond a critical value, $W_{s}$ starts to increase. The critical value of $E$ seems to depend on chain length; it decreases as chain length in-

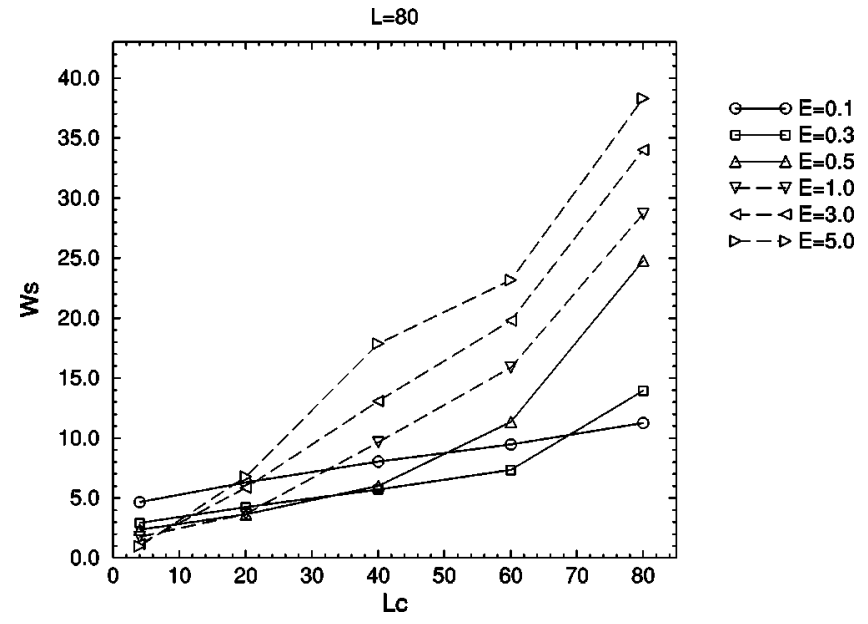

FIG. 8. Variation of saturated width $W_{s}$ with chain length $L_{c}$ at various field magnitudes, $E=0.1$ to $E=5.0$, in a lattice of size $L$ $=80$.

creases. Also, beyond the critical field, the saturated width increases with $E$ more rapidly as the chain length increases [Fig. 7(c)]. Rapid growth of the saturated width with the chain length is more transparent in a $W_{s}$ versus $L_{c}$ plot for different field strengths (see Fig. 8).

Chains of different length respond differently to the field and also vary in steric hindrance effects. This could account for the different effects seen in the field on the saturated width. Roughness of the polymer surface depends on packing of the chains in the bulk and on the surface. The packing density varies with the chain length with shorter chains having a higher packing density. Clearly, the field elongates and aligns the chains, and makes it easier for short chains to pack closer together. For the longer chains, the interplay between the field and steric effects is more complicated.

\section{SUMMARY AND CONCLUSION}

We used a simple model of polymer chains driven by an external field to study the growing surfaces as the chains deposit on an attractive wall. As in particle growth models, we see power-law growth of the polymer surfaces. The growth exponent $(\beta)$ depends on the strength of the driving field. At relatively low fields, two power-law regions are identified: exponents in the short time region are similar to those in a random deposition model, while in the long time, the exponents have higher values characteristic of driven (anisotropic) growth, due to a depinning influence of the field. Roughness of the polymer surface depends on packing of the chains in the bulk and on the surface, and depends strongly on the chain length and its conformational response to the field.

\section{ACKNOWLEDGMENTS}

Simulations were performed on Beowulf clusters at the Department of Computational Science of the National University of Singapore and at the University of Southern Mississippi. Partial support from a NSF-EPSCoR grant is acknowledged. 
[1] Dynamics of Fractal Surfaces, edited by F. Family and T. Vicsek (World Scientific, Singapore, 1991); A.-L. Barabasi and H. E. Stanley, Fractal Concepts in Surface Growth (Cambridge University Press, Cambridge, England, 1995).

[2] E. F. Edwards and D. R. Wilkinson, Proc. R. Soc. London, Ser. A 381, 17 (1982).

[3] M. Kardar, G. Parisi, and Y. C. Zhang, Phys. Rev. Lett. 56, 889 (1986).

[4] J. M. Lopez and H. J. Jensen, Phys. Rev. Lett. 81, 1734 (1998); F. Family, J. Phys. A 19, L441 (1986); D. E. Wolf and J. Villian, Europhys. Lett. 13, 389 (1990).

[5] H. Yan, Phys. Rev. Lett. 68, 3048 (1992); M. Kotrla, A. C. Levi, and P. Smilauer, Europhys. Lett. 20, 25 (1992); P. I. Tamborenea and S. Das Sarma, Phys. Rev. E 48, 2575 (1993).

[6] S. Das Sarma et al., Phys. Rev. B 49, 10693 (1994); J. M. Kim and S. Das Sarma, Phys. Rev. Lett. 72, 2903 (1994); C. Dasgupta, S. Das Sarma, and J. M. Kim, Phys. Rev. E 54, R4552 (1996).

[7] J. M. Kim and J. M. Kosterlitz, Phys. Rev. Lett. 62, 2289 (1989); J. M. Kim, J. M. Kosterlitz, and T. Ala-Nissila, J. Phys. A 24, 5569 (1991); K. Moser, J. Kertesz, and D. E. Wolf, Physica A 178, 215 (1991).

[8] P. Keblinski et al., Phys. Rev. E 53, 759 (1996); L. Golubovic and R. P. U. Karunasiri, Phys. Rev. Lett. 66, 3156 (1991).

[9] R. Bruinsma, in Surface Disordering Growth, Roughening and Phase Transitions, edited by R. Jullien, J. Kertesz, P. Meakin, and D. E. Wolf (Nova Science Publishers, New York, 1992), p. 91.

[10] K. Sneppen, Phys. Rev. Lett. 69, 3539 (1992); Luis A. Nunes Amaral, A.-L. Barabasi, and H. E. Stanley, ibid. 73, 62 (1994); S. Galluccio and Y.-C. Zhang, Phys. Rev. E 51, 1686 (1995); V. K. Horvath and H. E. Stanley, ibid. 52, 5166 (1995).

[11] H. Jeong, B. Kahng, and D. Kim, Phys. Rev. Lett. 77, 5094 (1996); H. Leschhorn, Phys. Rev. E 54, 1313 (1996); R. Albert et al., Phys. Rev. Lett. 81, 2926 (1998).

[12] R. P. Wool, Polymer Interfaces: Structure and Strength (Hanser Publishers, New York, 1995).

[13] P. G. de Gennes, Scaling Concepts in Polymer Physics (Cornell University, Ithaca, 1979).

[14] M. Doi and S. F. Edwards, The Theory of Polymer Dynamics (Clarendon, Oxford, 1986).

[15] Monte Carlo and Molecular Dynamics Simulations in Polymer Science, edited by K. Binder (Oxford University Press, New York, 1995).

[16] A. Baumgaertner, in The Monte Carlo Methods in Condensed Matter Physics, edited by K. Binder (Springer-Verlag, New York, 1995).

[17] Computational Modeling of Polymers, edited by J. Bicerano (Marcel Dekker, New York, 1992).

[18] G. M. Foo and R. B. Pandey, Phys. Rev. Lett. 79, 2903 (1997); J. Chem. Phys. 107, 10260 (1997); Phys. Rev. Lett. 80, 3767 (1998). 